\title{
NECESSIDADES DE CUIDADOS DOMICILIARES DE ENFERMAGEM APÓS A ALTA HOSPITALAR NO CONTEXTO DO SUS
}

\author{
Ana Carolina de Oliveira Jeronymo Neves ${ }^{1}$, Edna Aparecida Barbosa de Castro ${ }^{2}$, Sibely Rabaça Dias da Costa ${ }^{3}$
}

RESUMO: O artigo apresenta uma análise sobre as necessidades de cuidados domiciliares de enfermagem e o atendimento pelo Sistema Único de Saúde, após a alta, de adultos e idosos dependentes. Resulta de pesquisa realizada em duas etapas, entre outubro de 2012 e julho de 2013. A primeira, exploratória, com 217 pacientes internados para avaliar a capacidade de autocuidado, e demandas de cuidados após a alta, segundo constructo teórico de Orem. A segunda, com aporte da Teoria Fundamentada nos Dados, realizada em seis domicílios de pessoas com déficit de autocuidado, por meio de entrevista semiestruturada. Evidenciou-se dificuldades vivenciadas pelos pacientes e cuidadores no atendimento de suas necessidades, destacando-se a sobrecarga, a falta de apoio profissional e as dificuldades em atender a demanda por insumos e equipamentos. Conclui-se que a implantação efetiva da Atenção Domiciliar é imprescindível para a integralidade do cuidado.

DESCRITORES: Assistência domiciliar; Cuidados de enfermagem; Autocuidado; Política de saúde.

\section{NEEDS FOR AT-HOME NURSING CARE AFTER DISCHARGE FROM HOSPITAL IN THE SUS CONTEXT}

ABSTRACT: This article presents an analysis on the needs for at-home nursing care and care through the Unified Health System, after the discharge, of adults and dependent elderly. Research undertaken in two phases, between October 2012 and July 2013 . The first, exploratory research involved 217 patients hospitalized to assess the capacity for self-care and care demands after discharge according to Orem's theory. The second phase, based on the Grounded Theory, was developed at six homes of people with self-care deficit through semistructured interview. It was evidenced that the patients and caregivers evidenced difficulties in attending to their needs, highlighting the burden, lack of professional support and difficulties to respond to the demand for material and equipment. In conclusion, the effective implementation of Home Care is fundamental for care integrality.

DESCRIPTORS: Home nursing; Nursing care; Self-care; Health policy.

\section{NECESIDADES DE CUIDADOS DOMICILIARIOS DE ENFERMERÍA TRAS EL ALTA HOSPITALARIO EN EL CONTEXTO DEL SUS}

RESUMEN: El artículo presenta un análisis sobre las necesidades de cuidados domiciliarios de enfermería y la atención por el Sistema Único de Salud, tras el alta, de adultos y ancianos dependientes. Resulta de investigación desarrollada en dos etapas, entre octubre del 2012 y julio del 2013. La primera, exploratoria, con 217 pacientes internados para evaluar la capacidad de autocuidado, y demandas de cuidados tras el alta, según el constructo teórico de Orem. La segunda con base en la Teoría Fundamentada en los Datos, desarrollada en seis domicilios de personas con déficit de autocuidado, mediante entrevista semiestructurada. Fueron evidenciadas dificultades vividas por los pacientes y cuidadores en la atención a sus necesidades, destacándose la sobrecarga, la falta de apoyo profesional y las dificultades en atender a la demanda por insumos y equipos. Se concluye que la implantación efectiva de la Atención Domiciliaria es fundamental para la integralidad del cuidado.

DESCRIPTORES: Atención domiciliaria de salud; Atención de enfermería; Autocuidado; Política de salud.

${ }^{1}$ Enfermeira. Mestranda em Enfermagem. Universidade Federal de Juiz de Fora. Juiz de Fora, MG, Brasil.

${ }^{2}$ Enfermeira. Doutora em Saúde Coletiva. Docente de Enfermagem da Universidade Federal de Juiz de Fora. Juiz de Fora, MG, Brasil.

${ }^{3}$ Enfermeira. Mestre em Enfermagem. Enfermeira do Hemocentro Regional de Juiz de Fora. Juiz de Fora, MG, Brasil.

Autor Correspondente:

Ana Carolina de Oliveira Jeronymo Neves

Universidade Federal do Rio de Janeiro

R. Oswaldo Perlingeiro, lote 12 - 25770-390 - Petrópolis, RJ, Brasil

E-mail: anacarol.jeronymo@gmail.com
Recebido: $14 / 07 / 2016$

Finalizado: 25/10/2016 


\section{INTRODUÇÃO}

Este artigo apresenta uma investigação sobre as necessidades de cuidados domiciliares de enfermagem de adultos e idosos, após a alta hospitalar, considerando a capacidade de autocuidado parcial ou totalmente compensatória, de acordo com a teoria do autocuidado de Dorothea Orem.

A motivação para a pesquisa decorreu da participação das autoras no grupo de estudos e pesquisas sobre autocuidado e processo educativo em saúde e enfermagem da Universidade Federal de Juiz de Fora (GAPESE/UFJF). Ressaltam-se experiências vivenciadas pelas autoras no Projeto de Extensão "Consulta de enfermagem para o autocuidado após a alta hospitalar" e no Treinamento Profissional "Acompanhamento de enfermagem para o autocuidado após a alta hospitalar", realizados no Hospital Universitário (HU) da UFJF.

Acrescenta-se a participação na pesquisa multicêntrica "Atenção Domiciliar em saúde: efeitos e movimentos na oferta e demanda no Sistema Único de Saúde (SUS) no Estado de Minas Gerais", coordenada pelo Núcleo de estudos e pesquisas sobre ensino e prática de enfermagem da Universidade Federal de Minas Gerais (NUPEPE/UFMG). Estas experiências permitiram uma aproximação tanto teórica quanto prática da temática e a identificação de uma lacuna na produção científica acerca dos cuidados de enfermagem demandados em domicílio, após a Política Nacional de Atenção Domiciliar, reafirmada pela portaria 963, de 27 de maio de $2013^{(1)}$.

A Atenção Domiciliar (AD), forma de cuidado considerada "mais integral" e contínua, é percebida como um esforço de mudança do SUS, construindo-se à lógica da substitutividade, visando outros tipos de objetos e formas de cuidar ${ }^{(2-3)}$.

O cuidado domiciliar envolve a "casa da família", ambiente dinâmico, onde o enfermeiro ocupa-se da prestação de cuidados, da organização do processo de trabalho, da elaboração e gestão do plano de cuidados, juntamente com os demais membros da equipe ${ }^{(4)}$.

No domicílio, os cuidados demandados à enfermagem englobam atividades de menor densidade tecnológica, que requerem apoio-educação, as leve-duras, e as de maior densidade ${ }^{(5)}$.

Baseado na Resolução do Conselho Federal de Enfermagem (COFEN) n³58, o trabalho do enfermeiro torna-se fortalecido à medida que é pautado na Sistematização da Assistência de Enfermagem (SAE), permitindo o reconhecimento do que realmente é necessário para o cuidador e para o "ser cuidado", aumentando a resolubilidade dos cuidados demandados ${ }^{(6-7)}$.

O cuidado, como cerne epistemológico da enfermagem, sistematizado na troca de conhecimentos com outras ciências, constitui-se como objeto de estudo da teoria dos sistemas de enfermagem de Dorothea Orem, que defende a classificação do cliente em três subtipos. Os classificados como totalmente dependentes apresentam incapacidade total de desenvolver suas atividades de autocuidado; os parcialmente dependentes realizam determinadas atividades, sendo que as demais são realizadas pela enfermagem e/ou seus cuidadores; e os classificados como independentes devem ser apoiados, incluindo orientações à saúde ${ }^{(5)}$. O autocuidado, portanto, é a atividade que os indivíduos praticam em seu benefício para manter a vida, a saúde e o bem-estar ${ }^{(5)}$.

Para Carpenito-Moyet, em concordância com Orem, a Síndrome do Déficit do Autocuidado é compreendida como o estado em que o indivíduo apresenta função motora e cognitiva prejudicada, levando a diminuição da capacidade para a realização das atividades da vida diária (AVDs), que constituem ações básicas do cotidiano, como alimentar-se e deambular. Além disso, também envolve a diminuição no desenvolvimento das atividades instrumentais de vida diária (AIVDs), englobando, dentre outras ações, a capacidade de realizar compras e promover a manutenção do $\operatorname{lar}^{(8)}$. Assim, este diagnóstico de enfermagem contribui para a compreensão do processo de cuidar pela enfermagem na $A D$, junto a pacientes dependentes para o autocuidado.

Objetiva-se, portanto, apresentar uma compreensão das necessidades de cuidados de enfermagem e de suas formas de atendimento pelo SUS, no domicílio, após a alta, tomando por base a teoria do autocuidado de Orem. A análise busca dar ênfase em como são resolvidas as necessidades de cuidados domiciliares de enfermagem, pelos indivíduos com déficit para o autocuidado, destacando-se o atual cenário da AD. 
Pesquisa de natureza qualitativa, realizada com aporte metodológico da Teoria Fundamentada nos Dados (TFD), cujo objetivo foi desenvolver uma teoria substantiva sobre o fenômeno pesquisado, derivada de dados, reunidos e analisados sistematicamente. Conforme proposto por essa abordagem, utilizou-se o conceito de "amostragem teórica", na qual a amostragem está completa quando as teorias estão saturadas e desenvolvidas ${ }^{(9)}$.

Para o alcance dos objetivos a mesma foi desenvolvida em duas etapas. Uma exploratória, com coleta de dados nas enfermarias clínicas de um hospital de ensino, em Minas Gerais. Esta etapa visou a identificar o perfil dos usuários, a fim de captar participantes com demandas por cuidados de enfermagem domiciliares. Foram incluídos indivíduos maiores de dezoito anos, residentes no município sede da pesquisa e internados para tratamento clínico. Utilizou-se de dois formulários. Um para a organização do banco de dados, com a finalidade de registrar as internações e coletar dados nos prontuários. Outro, para a avaliação da capacidade do autocuidado, aplicado durante a visita de enfermagem, na forma da escala tipo Likert, desenvolvida com base nos referenciais de Orem ${ }^{(5)} \mathrm{e}$ Carpenito-Moyet ${ }^{(8)}$. Esta foi aplicada em 217 pacientes, entre outubro de 2012 e abril de 2013. Os dados coletados nesta fase foram organizados em um portfólio e submetidos à análise através de ferramentas estatísticas do programa Excel, contido no pacote da Microsoft.

Na segunda etapa, a pesquisa seguiu-se por meio de visitas domiciliares (VDs), incluindo-se como participantes os pacientes classificados, na fase anterior, em parcialmente ou totalmente dependentes. Os mesmos estavam orientados no momento da coleta de dados e tinham um cuidador disponível a participar, assinando o termo de consentimento.

Dos 217 pacientes avaliados, 93 eram potenciais participantes para a segunda etapa, sendo 71 parcialmente e 22 totalmente dependentes para o autocuidado. A coleta dos dados empíricos, na segunda fase, encerrou-se após visitas a seis participantes, dos quais quatro foram classificados como totalmente e dois como parcialmente compensatório, com demandas para os serviços de AD. Destes, três idosos (dois do sexo masculino e um feminino) e três adultos (dois do sexo feminino e um masculino).

Os dados foram coletados durante VD por meio de entrevistas, utilizando-se de roteiro semiestruturado, com duração média de duas horas. Cada entrevista era gravada e transcrita na íntegra, utilizando-se de pseudônimos para garantir o anonimato dos participantes. Antes da realização da entrevista seguinte, eram pré-analisadas e os dados sistematicamente codificados, com o auxílio do editor textual para dados qualitativos, OpenLogos (versão 2.0) ${ }^{(10)}$. Para agendamento das entrevistas estabeleceu-se contato telefônico. Esta fase da coleta de dados aconteceu entre maio e julho de 2013.

A análise dos dados seguiu os três tipos de codificação propostos por Strauss \& Corbin. Na codificação aberta as entrevistas foram examinadas minuciosamente para extrair os códigos, que foram agrupados, formando categorias; além disso, os conceitos foram identificados. Com o avanço da análise ocorreu a codificação axial, fase em que as categorias foram relacionadas às suas subcategorias. Na última fase aconteceu a codificação seletiva, onde buscou-se identificar a categoria central, validando sua relação com as demais categorias e dessas entre $\mathrm{si}^{(9)}$.

Essa pesquisa foi pautada nos aspectos éticos definidos pela Resolução $n^{\circ} 466$, de 12 de dezembro de $2012^{(11)}$. A coleta de dados foi iniciada após aprovação pelo Comitê de Ética em Pesquisa com Seres Humanos do HU/UFJF, sob $\mathrm{n}^{\circ} 229.712$.

\section{RESULTADOS}

Dos dados obtidos na etapa exploratória, verificou-se que os maiores números de internações foram ocasionados por neuropatias, 40 (18,43\%), seguida por doenças gastrointestinais, 33 (15,21\%), pneumopatias, $25(11,52 \%)$, doenças infecto-parasitárias, $24(11,06 \%)$, cardiopatias, $23(10,60 \%)$, nefropatias, $19(8,76 \%)$ e neoplasias, $18(8,29 \%)$. 
Verificou-se que o grau de dependência para o autocuidado apresentou estreita relação com o sexo e a faixa etária, conforme dados descritos nas Tabelas 1 e 2.

Tabela 1 - Distribuição dos pacientes internados nas enfermarias clínicas conforme avaliação da capacidade do autocuidado, sexo e faixa etária. Juiz de Fora, MG, Brasil, 2013

\begin{tabular}{|c|c|c|c|c|c|c|c|}
\hline \multirow{2}{*}{$\begin{array}{l}\text { Grau de } \\
\text { dependência }\end{array}$} & \multicolumn{2}{|c|}{ Adulto (<60anos) } & \multirow[t]{2}{*}{ Subtotal } & \multicolumn{2}{|c|}{ Idoso (>=60 anos) } & \multirow[t]{2}{*}{ Subtotal } & \multirow[t]{2}{*}{ Total } \\
\hline & Feminino & Masculino & & Feminino & Masculino & & \\
\hline Independente & $\begin{array}{c}30 \\
(50,85 \%)\end{array}$ & $\begin{array}{c}56 \\
(70,89)\end{array}$ & $\begin{array}{c}86 \\
(62,32 \%)\end{array}$ & $\begin{array}{c}15 \\
(42,86 \%)\end{array}$ & $\begin{array}{c}23 \\
(52,27 \%)\end{array}$ & $\begin{array}{c}38 \\
(48,10 \%)\end{array}$ & $\begin{array}{c}124 \\
(57,14 \%)\end{array}$ \\
\hline $\begin{array}{l}\text { Parcialmente } \\
\text { compensatório }\end{array}$ & $\begin{array}{c}22 \\
(37,29 \%) \\
\end{array}$ & $\begin{array}{c}17 \\
(21,52 \%) \\
\end{array}$ & $\begin{array}{c}39 \\
(28,26 \%) \\
\end{array}$ & $\begin{array}{c}16 \\
(45,71 \%) \\
\end{array}$ & $\begin{array}{c}16 \\
(36,36 \%) \\
\end{array}$ & $\begin{array}{c}32 \\
(40,51 \%) \\
\end{array}$ & $\begin{array}{c}71 \\
(32,72 \%) \\
\end{array}$ \\
\hline $\begin{array}{l}\text { Totalmente } \\
\text { compensatório }\end{array}$ & $\begin{array}{c}7 \\
(11,86 \%)\end{array}$ & $\begin{array}{c}6 \\
(7,59 \%)\end{array}$ & $\begin{array}{c}13 \\
(9,42 \%)\end{array}$ & $\begin{array}{c}4 \\
(11,43 \%)\end{array}$ & $\begin{array}{c}5 \\
(11,37 \%)\end{array}$ & $\begin{array}{c}9 \\
(11,39 \%)\end{array}$ & $\begin{array}{c}22 \\
(10,14 \%)\end{array}$ \\
\hline Total & 59 & 79 & 138 & 39 & 44 & 79 & 217 \\
\hline
\end{tabular}

Tabela 2 - Distribuição dos pacientes internados nas enfermarias clínicas, avaliados segundo a capacidade do autocuidado e sexo. Juiz de Fora, MG, Brasil, 2013

\begin{tabular}{lccc} 
Grau de dependência & Feminino & Masculino & \multirow{2}{*}{ Total } \\
\cline { 2 - 3 } & Adulto/Idoso & Adulto/Idoso & \\
\hline Independente & $45(47,87 \%)$ & $79(63,23 \%)$ & $124(57,14 \%)$ \\
\hline Parcialmente compensatório & $38(40,43 \%)$ & $33(26,83 \%)$ & $71(32,72 \%)$ \\
\hline Totalmente compensatório & $11(11,70 \%)$ & $11(8,94 \%)$ & $22(10,14 \%)$ \\
\hline Total & $\mathbf{9 4}(\mathbf{4 3 , 3 2} \%)$ & $\mathbf{1 2 3}(\mathbf{5 3 , 6 8} \%)$ & $\mathbf{2 1 7}$
\end{tabular}

Pelo processo de análise dos dados empíricos com a orientação da TFD obteve-se 25 códigos, distribuídos em três categorias, com suas respectivas subcategorias, conforme representado pelo Quadro 1.

Quadro 1 - Categorias e subcategorias. Juiz de Fora, MG, Brasil, 2013

\begin{tabular}{|l|}
\hline Binômio paciente-família no contexto domiciliar: vivências, aparências, percepções e sentimentos \\
\hline “Casa da família" na atenção domiciliar: aparências, personalidades e sentimentos \\
\hline Cuidados domiciliares: facilidades e dificuldades \\
\hline Categoria Central - O SUS na atenção domiciliar: oferta versus demanda por cuidados instrumentais \\
\hline Recursos humanos e a prestação de serviços no domicílio \\
\hline Rede de serviços: as necessidades por cuidados domiciliares e a (in)disponibilidade no SUS \\
\hline $\begin{array}{l}\text { O paciente e seu cuidador familiar na dependência para o autocuidado, e suas relações com as diversas } \\
\text { patologias }\end{array}$ \\
\hline Diagnósticos, patologias e internações: um novo olhar pela vida \\
\hline Déficit para o autocuidado versus cuidadores familiares \\
\hline A dependência por cuidados e suas implicações na vida cotidiana \\
\hline
\end{tabular}

Na categoria "Binômio paciente-família no contexto domiciliar: vivências, aparências, percepções e sentimentos", a religiosidade/espiritualidade surgiu de modo recorrente nos relatos.

A única coisa que eu tenho para falar é só agradecer, eu agradeço muito a Deus, porque estar viva e estar aqui ainda, graças a Deus, sem coisas piores na minha vida. Porque na verdade tudo passa. E eu creio que daqui a pouco, se Deus quiser eu posso está andando de novo. (Celeste) 
Além disso, a escassez de recursos financeiros foi informada como um obstáculo a ser enfrentado no atendimento dos cuidados domiciliares.

Estamos conseguindo porque a gente está pedindo ajuda. Igual as cadeiras de rodas a gente pediu. Fralda, ela já ganhou. O suplemento a gente está dividindo entre os irmãos, mas com dificuldade, porque a gente é assalariada, então, o dinheiro da gente não dá. (Luana)

Na categoria central denominada "O SUS na atenção domiciliar: oferta versus demanda por cuidados instrumentais", os participantes ressaltaram sentimentos de descaso pelos serviços de saúde para com os pacientes e/ou seus cuidadores, como relatado abaixo.

Eu acho assim, que o trabalho feito com o meu pai lá foi bom, mas na hora da alta foi como se tivesse descartando o meu pai a nosso cuidado. Agora daqui para frente é vocês e mais ninguém. (Ricardo)

Durante as entrevistas os participantes listaram necessidades de cuidados domiciliares e as dificuldades em atendê-las.

Ela está usando cadeira de rodas, está usando fraldas. (Luana)

Ah, já tem um ano que ele está com gastrostomia, mais ou menos isso. (Mariana)

E, que aí ficou agora com esse problema que tem que fazer, tem que usar o oxigênio 24 horas por causa da falta de ar. (Luana)

Já tentamos conseguir no SUS a fralda, mas é tanta burocracia que a gente desistiu para te falar a verdade. Eles tão, eles mandam, é assim. Aí ela vai lá e eles começam, pedem vários papéis de novo. (Luana)

Inserido na terceira categoria "O paciente e seu cuidador familiar na dependência para o autocuidado, e suas relações com as diversas patologias", destaca-se a sobrecarga física e psíquica do cuidador familiar, como relatado abaixo.

Porque eu tenho minha mãe também que está numa idade que dá muito trabalho. Assim esclerosada, fala coisas que às vezes magoa a gente. Ela não faz, eu faço tudo, sabe? Ás vezes sem aguentar, porque eu tenho problemas de estômago, ás vezes eu não estou nem aguentando, mas estou ali fazendo. (Mariana)

Ressalta-se, também, pelos pacientes sentimentos de medo e tristeza por continuarem incapazes para realizarem "simples" atividades da vida diária.

Mas eu acho que, andar mesmo, eu não vou conseguir. E não revolto, também, por causa disso. O que Deus está me dando eu tenho que aceitar. (Roberta)

\section{- DISCUSSÃO}

Pela etapa exploratória, analisa-se o perfil e a capacidade do autocuidado de pacientes que constituíram o cenário inicial da pesquisa, verificando-se que os maiores números de internação foram de pacientes do sexo masculino 123 (56,68\%). Este achado está em conformidade com estudos nacionais, voltados à análise do perfil das internações hospitalares ${ }^{(12-13)}$.

Quanto à capacidade do autocuidado, foram classificados em independentes para o autocuidado, em ambos os sexos e faixas etárias, $127(57,14 \%)$ pacientes. Entretanto, os pacientes do sexo masculino apresentaram maior grau de independência, 79 (64,23\%), quando comparados aos do sexo feminino. Assim, em discordância com estudo realizado em 2013, esta pesquisa encontrou diferença no grau de dependência relacionado ao gênero ${ }^{(14)}$.

Do total de idosos, verificou-se que 41 (51,9\%) apresentavam alguma dependência. Já nos pacientes adultos observou-se um menor percentual 52 (37,68\%). Estes dados estão em conformidade com um estudo nacional, que constatou relação direta entre idade e grau de dependência ${ }^{(14)}$.

Em relação às causas da internação hospitalar, a maior porcentagem de internações obtida foi por: neuropatias; seguida pelas doenças gastrointestinais; pneumopatias e infecto-parasitárias. 
De acordo com o atual estudo, uma pesquisa realizada na Zona da Mata Mineira destacou a maior incidência de pneumopatias, em segundo lugar as doenças gastrointestinais, e por último as neuropatias ${ }^{(14)}$.

Segundo dados mais recentes do DATASUS, publicados em 2008, têm-se que $13,76 \%$ dos pacientes são internados por cardiopatias com consequências neurológicas, em segundo lugar encontra-se as doenças gastrointestinais $(9,80 \%)$, seguidas pelas pneumopatias $(9,11 \%)$, em quarto lugar estão as doenças do aparelho geniturinário (7,56\%), seguida pelas neoplasias $(6,91 \%)$, as doenças infectoparasitárias $(5,81 \%)$, e por último estão os transtornos mentais $(3,34 \%)^{(15)}$.

\section{Binômio paciente-família no contexto domiciliar: vivências, aparências, percepções e sentimentos}

Esta categoria representa a relação existente entre o paciente e seu cuidador, no âmbito domiciliar, destacando-se aspectos vivenciados em cada cotidiano da "casa da família".

Nesse contexto domiciliar, destaca-se à Divindade como principal fonte de suporte para suas vidas, depositando-se a Esta as esperanças de superação da elevada carga de cuidados.

A necessidade de proporcionar um tratamento mais completo e benéfico, por meio da compreensão da complexidade e da importância do papel do orientador espiritual foi evidenciada numa pesquisa realizada nos Estados Unidos ${ }^{(16)}$.

Nota-se que o apoio ao autocuidado por meio do amparo à religiosidade/espiritualidade contribui para a aderência à terapêutica, redução do tempo de tratamento e à tomada de decisões ${ }^{(17)}$.

Como dificuldades a serem superadas pela família, destaca-se a escassez de recursos financeiros, levando à busca de alternativas para a prestação do cuidado delegado pelo SUS.

Corrobora-se com achados de outros estudos que no cotidiano das famílias atendidas pela AD identificam-se dificuldades de ordens econômicas e sociais, como desemprego e o alto custo do tratamento ${ }^{(18-19)}$.

É papel do Estado a ampliação do acesso à AD, com a prestação de cuidados de qualidade, de forma que não seja transferida aos cuidadores toda a responsabilidade pelo paciente, tanto na execução dos cuidados quanto na transferência de gastos ${ }^{(1)}$. Os gastos elevados com serviços de AD, realizados pelos próprios familiares, podem ameaçar a economia da família e o bem-estar do paciente.

\section{O SUS na Atenção Domiciliar: oferta versus demanda por cuidados instrumentais}

Esta categoria apresenta uma análise sobre as necessidades de cuidados de enfermagem, temática que se destaca no cenário atual das políticas públicas relacionadas à AD.

De acordo com relato dos participantes da pesquisa, verificou-se ausência de realização sistemática, por enfermeiros, da educação terapêutica para a alta. Ao relatarem recebimento de orientações, independente da categoria profissional, os participantes enfatizaram a falta de qualidade, mantendoos sem entender o que e como fazer, para o alívio das inseguranças e, sobretudo, para a resolubilidade das necessidades de saúde.

Diante disso, entende-se que o preparo para alta precisa ser planejado desde a admissão, para que não haja sobrecarga de informações no momento da saída ${ }^{(13)}$.

As orientações precisam ser realizadas de forma sistemática e vinculadas a rede de atenção à saúde (RAS), com vistas à integralidade. Porém, sabe-se que a RAS ainda é um processo em construção, com lacunas importantes referentes a práticas fragmentadas ${ }^{(20)}$.

Na busca por diagnósticos e tratamentos, representados por idas e vindas, os pacientes e seus cuidadores relatam que se sentem "perdidos", "engolidos pelo sistema", ou seja, desorientados na busca por resolução de seus problemas. Esta situação aponta para uma possível elevação da demanda de cuidados domiciliares, superior a capacidade de atendimento das equipes de AD existentes, ou seja, uma relação desequilibrada entre oferta e demanda ${ }^{(2)}$.

A partir de relatos intensos e recorrente dos participantes, compreende-se a importância da oferta de cuidados de enfermagem domiciliares, por vezes delegados ao paciente e a família, sem, todavia, 
uma avaliação profícua e sistemática de que tenham condições de realizá-lo.

Um estudo atual sobre as necessidades de cuidados domiciliares mostrou que: $73,7 \%$ dos pacientes usavam dieta enteral, $24,6 \%$ faziam uso de traqueostomia, 22,8\% necessitavam de oxigenoterapia, $17,5 \%$ realizavam aspiração de vias aéreas, $8,7 \%$ monitorizavam a glicemia capilar e 5,2 \% realizavam diálise peritoneal ${ }^{(21)}$.

\section{O paciente e seu cuidador familiar na dependência para o autocuidado, e suas relações com as diversas patologias}

A pesquisa identificou a vivência de cuidados na esfera das AVDs e AIVDs, realizados pelos próprios pacientes e seus cuidadores. Vivência esta, envolta por sentimentos próprios do processo de adoecimento e do grau de dependência.

Sabe-se que, no contexto do cuidado domiciliar, o paciente e seus familiares vivem sentimentos intensos, não apenas pela proximidade da morte, mas, também, pela conscientização de uma doença que o coloca em risco à vida ${ }^{(22)}$.

O adoecimento do familiar muda a rotina da família, aumenta as responsabilidades e dificuldades, altera o modo de ver e viver à vida. Para o cuidador essa mudança é mais intensa. Este se torna sobrecarregado, com um turbilhão de sentimentos, porém, ao mesmo tempo se sente culpado, tenta mostrar ao familiar doente e a sociedade que ele é forte, que consegue fazer o melhor para o seu ente.

Diante desse processo de adoecimento e dependência, os cuidadores destacam sobrecarga física e psíquica relacionados ao aumento da demanda de tempo e das despesas com o cuidado $^{(23)}$.

Dentro desta categoria, ressalta-se, também, a relação entre a evolução da patologia e o seu grau de dependência, onde a perda da autonomia e o comprometimento de funções que incluam a realização de AVDs modificam o cotidiano do indivíduo, gerando sentimentos de frustração, tristeza e impotência ${ }^{(24)}$.

À medida que o paciente e sua família vivenciam esta nova situação, de dependência e incapacidade, a estrutura física e funcional da "casa da família" sofre reajustes e tem que ser adaptada, por vezes de modo brusco.

Teoria geral do estudo: "Necessidades de cuidados domiciliares de enfermagem após a alta hospitalar no contexto do SUS"

Mediante a metodologia utilizada neste estudo, tem-se como resultado final uma teoria derivada nos dados, sintetizada no diagrama a seguir.

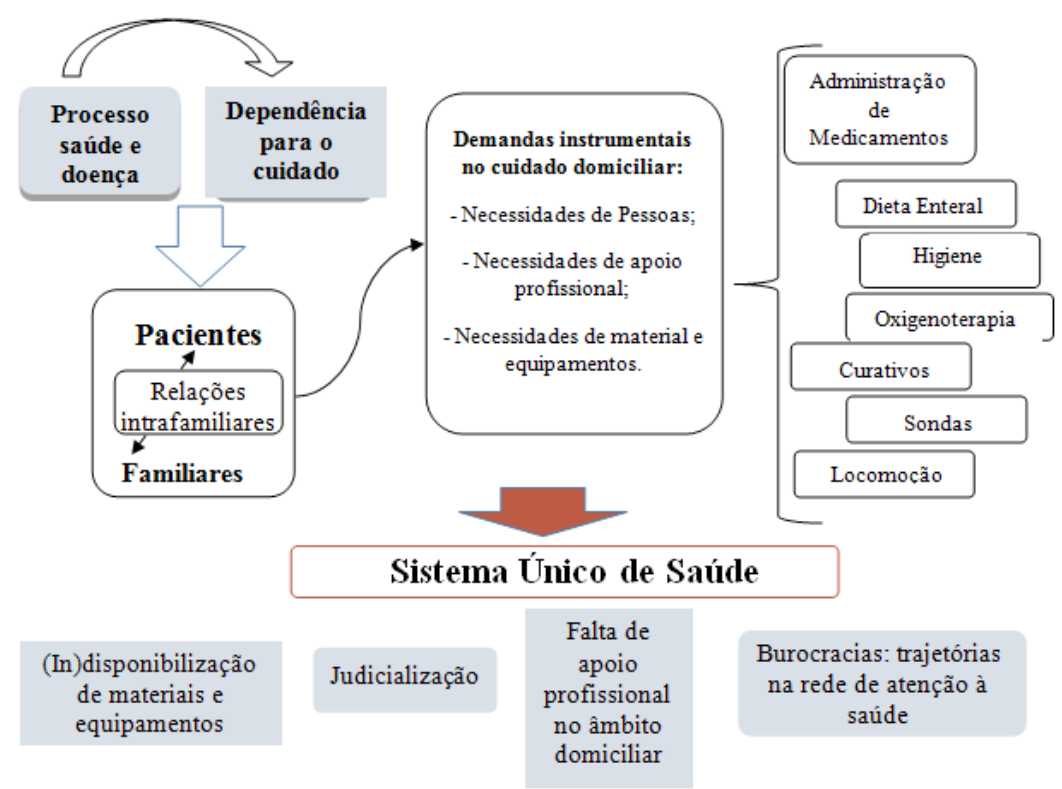

Diagrama 1 - Teoria geral do estudo "Necessidades de cuidados domiciliares de enfermagem após a alta hospitalar no contexto do SUS". Juiz de Fora, MG, Brasil, 2013 
Pela análise em profundidade, pode-se compreender que no decorrer do processo de adoecimento secundário a doenças crônico-degenerativas, os participantes desenvolveram um grau de dependência, classificados em parcialmente ou totalmente dependentes de cuidados domiciliares. A provisão dos cuidados torna-se mais complexa à medida que envolve o binômio paciente-família, em um determinado contexto social, econômico e político.

O surgimento da patologia, com comprometimento da capacidade para o autocuidado, coloca em evidência os conflitos e as relações intrafamiliares conturbadas. Ao buscarem suprir as demandas de cuidados domiciliares, decorrentes do grau de dependência, o usuário do SUS, juntamente com o cuidador familiar, inicia um processo de "idas e vindas". Nessas "idas e vindas", com sofrimento ativo, investem, tentam, persistem até "conseguir", ou não, o atendimento de suas necessidades por cuidados instrumentais e terapêuticos, que neste estudo representam-se por: oxigenoterapia, higiene corporal, nutrição, curativos, mobilidades, medicamentos e sondagens. Para a resolubilidade destes cuidados, os familiares enfrentam burocracias e o que chamam de "falhas" do SUS, evidenciadas por falta de apoio profissional no âmbito domiciliar, indisponibilidade de materiais e de equipamentos. Para a obtenção, alguns usuários lançam mão do mandato judicial.

\section{CONCLUSÃo}

A pesquisa desperta a enfermagem brasileira para um novo cenário de atenção à saúde, sinalizando à necessidade de formação e capacitação para a atuação na AD. As dificuldades apontadas na busca por cuidados no SUS giram em torno de necessidades que encontram-se no campo de atuação, demandando ações na área da gestão do cuidado, da assistência, da formação e realização de pesquisas.

Considerando-se o porte do hospital sede da pesquisa e suas especificidades no SUS, os resultados podem não expressar a realidade de outras localidades. Sendo assim, ressalta-se a necessidade de realização de outras pesquisas, sobre esta temática, aprofundando as buscas em outras regiões geográficas.

Concluiu-se que é relevante a ampliação do processo de implantação da $\mathrm{AD}$, conforme desencadeada pelo vigente Programa Melhor em Casa, a fim de superar "aborrecimentos e tormentos" de usuários e famílias fragilizados, imersos em um universo de necessidades de cuidados domiciliares contínuos.

\section{REFERÊNCIAS}

1. Brasil. Portaria n. 963, de 27 de maio de 2013. Redefine a Atenção Domiciliar no âmbito do Sistema Único de Saúde (SUS). Diário Oficial da União, n. 101, Brasília, 28 mai. 2013. Seção 1:30.

2. Andrade AM, Brito MJM, Silva KL, Von Randow RM, Montenegro LC. Singularidades do trabalho na Atenção Domiciliar: imprimindo uma nova lógica em saúde. R. pesq.: cuid. fundam. online. [Internet] 2013;5(1) [acesso em 12 fev 2016]. Disponível: http://dx.doi.org/10.9789/2175-5361.2013v5n1p3383.

3. Silva KL, de Sena RR, Seixas CT, Feuerwerker LCM, Merhy EE. Atenção domiciliar como mudança do modelo tecnoassistencial. Rev. Saúde Pública. [Internet] 2010;44(1) [acesso em 27 fev 2016]. Disponível: http://dx.doi. org/10.1590/S0034-89102010000100018.

4. Silva KL, de Sena RR, Silva PM, Braga PP, de Souza CG. Serviços de atenção domiciliar na saúde suplementar e a inserção da enfermagem em Belo Horizonte/MG. Acta paul. enferm. [Internet] 2012;25(3) [acesso em 1 dez 2015]. Disponível: http://dx.doi.org/10.1590/S0103-21002012000300014.

5. Orem DE. Nursing: concepts of practice. New York: McGraw-Hill; 1985.

6. Pinto EA, da Silva DDA, dos Santos RM, Trezza MCSF. Necessidades de cuidados expressas pela família que possui um acamado no domicílio. Enferm Foco. [Internet] 2012;3(4) [acesso em 6 fev 2016]. Disponível: http:// revista.portalcofen.gov.br/index.php/enfermagem/article/view/383.

7. Conselho Federal de Enfermagem (COFEN). Resolução n. 358, de 15 de outubro de 2009. Dispõe sobre a 
Sistematização da Assistência de Enfermagem e a implementação do Processo de Enfermagem em ambientes, públicos ou privados, em que ocorre o cuidado profissional de Enfermagem, e dá outras providências. Rio de Janeiro: COFEN; 2009.

8. Carpenito-Moyet LJ. Diagnósticos de Enfermagem: Aplicação à Prática Clínica. 13ª ed. Porto Alegre: Artmed; 2012.

9. Strauss A, Corbin J. Pesquisa Qualitativa: técnicas e procedimentos para o desenvolvimento de teoria fundamentada. $2^{a}$ ed. Porto Alegre: Artmed; 2008.

10. Camargo Junior, KR. Apresentando Logos: um gerenciador de dados textuais. Cad. Saúde Pública. [Internet] 2000;16(1) [acesso em 05 dez 2015]. Disponível: http://www.researchgate.net/publication/282651258_ Apresentando_Logos_Um_gerenciador_de_dados_textuais.

11. Ministério da Saúde (BR). Conselho Nacional de Saúde. Diretrizes e normas regulamentadoras de pesquisa envolvendo seres humanos. Resolução n. 466, de 12 de dezembro de 2012. Brasília; 2012.

12. Nogueira LS, de Sousa RMC, Padilha KG, Koike KM. Características clínicas e gravidade de pacientes internados em UTIs públicas e privadas. Texto Contexto Enferm. [Internet] 2012;21(1) [acesso em 2 fev 2016]. Disponível: http://dx.doi.org/10.1590/S0104-07072012000100007.

13. Souza ICP, Silva AG, Quirino ACS, Neves MS, Moreira LR. Perfil de pacientes dependentes hospitalizados e cuidadores familiares: conhecimento e preparo para as práticas do cuidado domiciliar. REME, Rev. Min. Enferm. [Internet] 2014;18(1) [acesso em 2 fev 2016]. Disponível: http://www.dx.doi.org/10.5935/1415-2762.20140013.

14. da Costa SRD, de Castro EAB, Acioli S. Capacidade de autocuidado de adultos e idosos hospitalizados: implicações para o autocuidado de enfermagem. REME, Rev. Min. Enferm. [Internet] 2013;17(1) [acesso em 25 nov 2015]. Disponível: http://dx.doi.org/10.5935/1415-2762.20130016.

15. Ministério da Saúde (BR). Informações de saúde. Departamento de Informática do SUS-DATASUS. [Internet] 2016 [acesso em 15 mar 2016]. Disponível: http://tabnet.datasus.gov.br/cgi/tabcgi.exe?idb2008/d13.def.

16. Lillis BS. Understanding the complex role of a hospice spiritual counselor. Am J Hosp Palliat Care. [Internet] 2014;31(4) [acesso em 11 fev 2016]. Disponível: http://dx.doi.org/10.1177/1049909113494746.

17. Santo CCE, Gomes AMT, de Oliveira DC, Marques SC. Adesão ao tratamento antirretroviral e a espiritualidade de pessoas com HIV/AIDS: estudo de representações sociais. Rev. enferm. UERJ. [Internet] 2013;21(4) [acesso em 02 mar 2016]. Disponível: http://www.e-publicacoes.uerj.br/index.php/enfermagemuerj/article/view/10005/7801.

18. Freitas IBA, Meneghel SN, Selli L. A construção do cuidado pela equipe de saúde e o cuidador em um programa de atenção domiciliar ao acamado em Porto Alegre (RS, Brasil). Ciênc. saúde coletiva. [Internet] 2011;16(1) [acesso em 17 nov 2015]. Disponível: http://dx.doi.org/10.1590/S1413-81232011000100032.

19. Janus AL, Ermisch J. Who pays for home care? A study of nationally representative data on disabled older Americans. BMC Health Serv Res. [Internet] 2015;(15) [acesso em 11 fev 2016]. Disponível: http://bmchealthservres. biomedcentral.com/articles/10.1186/s12913-015-0978-x.

20. Brito MJM, Andrade AM, Caçador BS, Freitas LFC, Penna CMM. Atenção domiciliar na estruturação da rede de atenção à saúde: trilhando os caminhos da integralidade. Esc. Anna Nery. [Internet] 2013;17(4) [acesso em 05 dez 2015]. Disponível: http://dx.doi.org/10.5935/1414-8145.20130002.

21. Brondani CM, Ramos LH, Beuter M, Lampert MA, Seiffert MA, Bruinsma JL. Caracterização de pacientes dependentes de tecnologias de um serviço de internação domiciliar. Rev. Enferm. UFSM. [Internet] 2013;3(n. esp) [acesso em 30 jan 2016]. Disponível: http://dx.doi.org/10.5902/2179769211063.

22. Rodriguez MIF. Internação domiciliar: avaliações imperativas implicadas na função de cuidar. Psic. Rev. [Internet] 2013;22(2) [acesso em 20 jan 2016]. Disponível: http://revistas.pucsp.br/index.php/psicorevista/article/ view/17989.

23. da Costa SRD, de Castro EAB. Autocuidado do cuidador familiar de adultos ou idosos dependentes após a alta hospitalar. Rev. bras. enferm. [Internet] 2014;67(6) [acesso em 14 nov 2015]. Disponível: http://dx.doi. org/10.1590/0034-7167.2014670617. 
24. Faber V, Rosanelli CP, Loro MM, Kolankiewicz ACB, Piovesan S, Leite MT. Percepção de doentes crônicos acerca do cuidado prestado por familiares. Cienc Cuid Saude. [Internet] 2012;11(3) [acesso em 09 fev 2016]. Disponível: http://dx.doi.org/10.4025/cienccuidsaude.v11i3.14990. 